\title{
Philonsorbonne
}

8 | 2014

Année 2013-2014

\section{Pouvoir et violence. Éléments pour une critique arendtienne de l'espace public habermassien}

\section{Clotilde NOUËT}

\section{(2) OpenEdition \\ Journals}

Édition électronique

URL : https://journals.openedition.org/philonsorbonne/605

DOI : 10.4000/philonsorbonne.605

ISSN : $2270-7336$

Éditeur

Publications de la Sorbonne

\section{Édition imprimée}

Date de publication : 1 janvier 2014

Pagination : 167-176

ISSN : 1255-183X

\section{Référence électronique}

Clotilde NOUËT, «Pouvoir et violence. Éléments pour une critique arendtienne de l'espace public habermassien », Philonsorbonne [En ligne], 8| 2014, mis en ligne le 19 janvier 2014, consulté le 08 juin 2021. URL : http://journals.openedition.org/philonsorbonne/605 ; DOI : https://doi.org/10.4000/ philonsorbonne.605 


\section{Pouvoir et violence. \\ Éléments pour une critique arendtienne de l'espace public habermassien}

\section{Clotilde NouËT}

Selon Habermas, l'Aufklärung constitue une rupture historique décisive, dans la mesure où elle promeut un rapport inédit - politique, à l'exercice de la raison. Le clivage instauré par l'État moderne, entre l'ordre public du pouvoir et la sphère privée de la croyance, de l'opinion et de la conscience morale, qu'entérine un penseur comme Hobbes, explique que chez Bayle, la critique demeure encore une affaire privée. L'émergence de l'opinion publique signe au contraire l'apparition, sur la scène même du pouvoir politique, d'un usage collectif de la raison sous la forme de discussions et d'échanges argumentés. Matrice d'une dissolution discursive de la domination, la sphère publique (Öffentlichkeit) se constitue autour de la détermination d'un intérêt général au-delà des particularismes. Tant que la raison d'État garantit l'ordre public, les intérêts subjectifs sont exclus de la scène politique. Mais dès lors que le public revendique, à travers la pratique de l'auto-législation, le droit de participer aux affaires de la communauté, se pose le problème de l'articulation entre la sphère de l'autonomie privée et celle de la citoyenneté. Repérée initialement dans la théorie rousseauiste de l'aliénation entre l'homme et le citoyen, cette question est reconduite jusque dans Droit et démocratie 1 , à l'occasion d'une réflexion sur l'articulation entre autonomie privée et publique, entre la liberté d'action subjective et les droits politiques de participation et de communication. Pour autant, l'égalitarisme universaliste que revendique Habermas ne se fonde pas sur l'hypothèse libérale d'une autonomie de la sphère politique, justifiant que la sphère des échanges sociaux, et donc économiques, se soustraie à la 
thématisation. Au contraire, dès 1962, l'universalisme abstrait du principe kantien de Publizität s'articule, au-delà de la critique hégélienne de «l'opinion publique » libérale, à la critique marxiste de l'émancipation politique développée dans la Question juive. Habermas y distingue deux moments : tout d'abord, la dénonciation d'un ordre bourgeois fondé sur la contradiction entre les principes juridiques normatifs des droits de l'homme et une structure socio-économique de classe qui, sous l'apparence de la liberté contractuelle, neutralise les rapports de pouvoir sous-jacents. Ensuite, articulée à la critique idéologique, la possibilité d'une subversion de la sphère publique bourgeoise conduit nécessairement au renversement de son fondement.

Mais selon lui, le contre-modèle socialiste marxiste opérerait sur la base d'une simple inversion ${ }^{2}$ de l'idéologie bourgeoise. Qu'une communauté soit fondée sur l'autonomie publique de ses membres n'est pas mis en question. Cette lecture dénote le souci de préserver le modèle universaliste qui sous-tend l'idée de l'espace public. À l'occasion de sa confrontation avec la féministe Carol Pateman, Habermas reconnaît la structure sexuée de la sphère publique, telle qu'elle s'est historiquement constituée ; mais il maintient l'existence, en amont, d'un fondement « plus profond ${ }^{3} »$.

La sphère publique bourgeoise s'articule sur des discours auxquels non seulement le mouvement ouvrier, mais aussi son autre exclu, donc le mouvement féministe, pouvait se rattacher pour les transformer de l'intérieur mais aussi pour transformer les structures de la sphère publique elle-même. Les discours universalistes de la sphère publique bourgeoise étaient placés, dès l'origine, sous des prémisses auto-référentielles; ils ne pouvaient rester immunisés contre une critique interne ${ }^{4}$.

Habermas postule ainsi un partage entre le fondement discursif normatif qui se trouve en amont des rapports sociaux de pouvoir, et les relations effectives de domination susceptibles d'être dissoutes de façon immanente. Un tel modèle n'est capable de théoriser l'existence de « contre-publics ${ }^{5}$ » ou

2. J. Habermas, L'espace public, 1962, rééd. 2010, Paris, Payot (avec la Préface de 1990), p. 137: «Marx tire de la dialectique immanente à la sphère publique bourgeoise les conséquences qui lui permettent de proposer un contre-modèle socialiste où le rapport traditionnel entre sphères publique et privée s'inverse de façon particulière. [...] L'autonomie ne peut plus y avoir pour base la propriété privée ; il est tout à fait impossible alors qu'elle ait encore pour assise la sphère privée : c'est au sein de la sphère publique elle-même que l'autonomie doit être fondée. Dès lors, l'autonomie privée n'est pas autre chose qu'une conséquence de cette autonomie première que le public des citoyens instaure par le seul exercice des fonctions propres à la sphère publique et dont l'éventail s'est élargi au sein du modèle socialiste. Un public de personnes privées fait face aux personnes privées d'un public ».

3. Ibid., préface, XXI.

4. Ibid., préface, IX.

5. O. Negt (L'espace public oppositionnel) défend l'idée d'une Gegenöffentlichkeit prolétarienne. 
de «publics subalternes ${ }^{6} »$ qu'à partir du fond commun où se lit leur opposition à la sphère publique bourgeoise. Derrière tout espace public historique, se trouve le principe normatif de la publicité qui subsume les structures politiques d'une intersubjectivité matricielle, relevant d'une pratique communicationnelle. Il faut donc faire retour sur la théorie de la communication et sa déclinaison proprement politique. Un article de 1976 nous semble en constituer la charnière, dans lequel, à l'occasion d'une confrontation décisive avec la pensée d'Hannah Arendt, Habermas interroge la genèse du «pouvoir communicationnel» caractéristique de l'espace public.

(I) En plaçant l'accent sur un texte où s'élabore concrètement cette notion, nous cherchons à éclairer le champ théorique où Habermas a inscrit sa compréhension politique du discours. Il apparaît en effet que la récupération du concept de pouvoir à laquelle il procède est permise par un véritable coup de force, qui l'empêche de saisir la spécificité de l'agencement théorique arendtien.

(II) En réactualisant le champ conceptuel arendtien, nous cherchons à faire jouer, au sein du paradigme habermassien, un élément d'extranéité qu'il recouvre - ou refoule. L'enjeu consiste à reformuler la question de la rationalité discursive sous-jacente à l'espace public, en l'articulant au problème politique de la violence.

(I) L'article «Hannah Arendts Begriff der Macht ${ }^{7} »$ se déploie selon deux moments décisifs.

1. Habermas commence par interpréter le pouvoir arendtien dans les termes d'un acte de langage illocutoire, pour aussitôt dénoncer sa base théorique. Ainsi, la notion chez Arendt recouvrirait la seule pratique de l'interaction visant l'entente, au cours de laquelle les participants délaissent leurs intérêts privés au profit de l'action commune : intuition intéressante, qui permet de contrer une interprétation strictement téléologique de l'action, représentée principalement par Weber. Toutefois, elle ne permettrait pas de prendre en charge la dimension stratégique de certaines interactions, par exemple la compétition individuelle pour le pouvoir. Habermas interroge ici le concept arendtien sur la base d'un partage discutable ${ }^{8}$. Mais en ouvrant

6. N. Fraser, «Repenser la sphère publique : contribution à une critique de la démocratie réellement existante », in E. Renault, Y. Sintomer (dir.), Où en est la théorie critique ?, Paris, La Découverte, 2003.

7. J. Habermas, Politisch-philosophische Profile, Francfort, Suhrkamp, 1981. L'article a été traduit en anglais, mais à notre connaissance pas en français.

8. Cette distinction entre la dimension stratégique et communicationnelle de l'action n'est pas pertinente chez Arendt, qui pense au contraire que l'exercice collectif du pouvoir repose sur la détermination d'un intérêt commun, qui guide le cours de l'action et lui impose des « objectifs déterminés ». (C'est d'ailleurs ce qui distingue l'action politique de l'idéologie en acte, qui suppose au contraire une absence totale de prise en compte des intérêts concrets d'une collectivité mais s'articule sous la forme d'un discours d'une cohérence absolue.) Voir entre 
le concept «illocutoire» du pouvoir au faisceau des «interactions stratégiques », il s'agit de souligner la présence, au sein même de la cité, des luttes de pouvoir; d'introduire également, par le biais de l'institutionnalisation de l'action stratégique (ou téléologique), à la considération d'une rationalité autre, la rationalité instrumentale.

2. Ainsi, le pouvoir chez Arendt, ancré dans une sphère du politique qui exclurait ontologiquement la sphère socio-économique de la production et des échanges, ni ne prendrait en compte la violence structurelle encastrée dans les institutions, demande à être interprété sur une base plus large. Habermas fait signe ici vers la distinction entre deux types de rationalité, communicationnelle et systémique, permettant de faire droit à la double dimension culturelle et symbolique de la légitimité et la dimension non normative du médium qu'est le pouvoir politique. L'action à proprement parler se situe au niveau fonctionnel de l'appareil d'État. Le concept du politique selon Habermas se saisit à la jonction de deux sphères institutionnelles ${ }^{9}$, la première productrice de normes, la seconde régulée fonctionnellement.

****

Cette première élaboration $d u$ «pouvoir communicationnel » propre à l'espace public, qui explique son rôle central dans la théorie politique habermassienne, sera complétée de deux apports significatifs. Tout d'abord, la Théorie de l'agir communicationnel (1981) permet de reconstituer la pragmatique formelle de cette situation de parole idéale que présuppose déjà l'article de 1976 (1). Ensuite, dans Droit et démocratie (1992), il s'agit de thématiser le rapport entre les cycles communicationnels de l'espace public et les procédures mises en œuvre par l'institution de l'État de droit (2). C'est dans l'articulation entre les structures de la rationalité discursive à proprement parler et l'espace empirique où celles-ci se déploient que se situe le noyau du « pouvoir communicationnel ».

(1) Deux types d'idéalisations, contrefactuelles, peuvent être repérées en amont d'un acte de langage : une idéalisation sémantique, c'est-à-dire la présupposition d'un langage commun permettant le rapport à un monde ; un système de prétentions à la validité intersubjective, qui règle le rapport entre les interlocuteurs ${ }^{10}$. L'enjeu consiste à établir, derrière tout rapport discursif,

autres, «Le totalitarisme », in Les origines du totalitarisme, Paris, Gallimard, Quarto, 2002, p. 634 et suivantes.

9. C'est l'objet de la critique de N. Fraser dans « What's Critical about Critical Theory ? The Case of Habermas and Gender », New German Critique, n³5, printemps-été 1985: cette distinction n'est pas uniquement méthodologique, elle renvoie bien à un partage institutionnel qui fait correspondre l'espace public et l'État, administré par le médium du pouvoir ; la sphère privée et le marché, régulé par le médium de l'argent.

10. « À travers son acte de parole, le locuteur cherche à s'entendre à propos de quelque chose avec son auditeur. Cette fin, qu'on peut appeler illocutoire, comporte deux niveaux : d'abord, l'auditeur doit comprendre l'acte de parole, puis, autant que possible, l'accepter». J. Habermas, Vérité et justification, Paris, Gallimard, 2001 [1998], p. 51. 
la normativité immanente que recueille le langage ${ }^{11}$, et qui se ressaisit au niveau d'une pragmatique formelle capable de penser la structure et le jeu, l'espace de liberté et de subversion que celle-ci autorise ${ }^{12}$. Ainsi, les structures communicationnelles ne sont pas directement contraignantes. Elles ne prescrivent aucun contenu normatif, seule leur forme constitue une «contrainte transcendantale faible ${ }^{13}$ », du fait qu'elle renvoie à une rationalité universelle, mais non déterminée par une forme de vie substantielle. Par ailleurs, c'est bien parce que l'on suppose une situation de parole idéale que l'on est en mesure d'évaluer les communications pathologiques ou déformées. La critique des blocages structurels (idéologies) ou contingents (manipulation), opère nécessairement à partir d'un critère, qu'il s'agit précisément d'expliciter en termes contrefactuels.

(2) Cette relecture communicationnelle de l'espace public permet de reformuler le problème classique de la légitimité du pouvoir. Récusant l'alternative entre le caractère irréductiblement positif, voire décisionniste du pouvoir, ou la nécessité de le fonder sur des droits naturels, Habermas cherche à penser ensemble son immanence normative, garantie par la structure d'universalité de la rationalité discursive, et son absolue spontanéité. Et c'est cette double perspective que lui semble autoriser, après révision, la praxis anarchique dont Arendt aurait fait l'essence de la politique. Ainsi, l'espace public joue un rôle opératoire dans la théorie habermassienne: constitué par un élément d'indisponibilité irréductible, il offre une possibilité de résistance ultime à la «colonisation du monde vécu ${ }^{14} »$. Face au monde administré, existe la source vive, et non domestiquée, de la communication : «On ne peut [...] contraindre une population à la spontanéité, fût-ce par le droit ${ }^{15} »$. L'espace public finit de fait par s'étendre à la totalité d'une société, nébuleuse communicationnelle anarchique qui offre un espace de thématisation illimité, toutefois polarisé, à travers le droit, jusqu'au niveau du système politique, par un jeu de procédures revêtant le double caractère de la positivité et de la norme immanente aux discussions argumentées. Dans les textes politiques les

11. Cela ne signifie pas, bien sûr, que langage et rationalité communicationnelle soient identiques, mais n'empêche pas, toutefois, que la structure normative de l'intersubjectivité soit ressaisie à partir d'un usage déterminé (pragmatique) du langage. Nous ne sommes pas en mesure de développer ce point ici, mais renvoyons à J. Habermas, Vérité et justification, op. cit., p. 70 : «Quel rapport y a-t-il entre le langage en tant que tel et la rationalité des opinions, des actions, des actes de communication et des personnes?».

12. «Dans le cadre de la théorie de l'agir communicationnel, je m'attache [...] à établir la plausibilité du fait qu'une personne, socialisée dans quelque langue et dans quelque forme culturelle que ce soit, ne peut pas ne pas s'engager dans des pratiques communicationnelles et, par là même, ne peut pas ne faire fond sur certaines présuppositions pragmatiques », J. Habermas, L'éthique de la discussion et la question de la vérité, Paris, Grasset, 2003, p. 29 (nous soulignons).

13. J. Habermas, Droit et démocratie, op. cit. p. 18.

14. Thèse dont l'article de 1976 ne fait pas encore état. Voir Théorie de l'agir communicationnel, Paris, Fayard, 1987 [1981), p. 392 et suivantes.

15. J. Habermas, Droit et démocratie, op. cit., p. 149. 
plus tardifs, il s'agit bien là d'un élément crucial, la "charnière » entre deux ensembles de systèmes ${ }^{16}$ (l'État administratif, la société capitaliste), renvoyant à cette société qui, s'auto-organisant par le biais des médiums fonctionnels ${ }^{17}$, est «devenue politique en elle-même» - formulation de Marx que Habermas revendique dans sa préface de 1990. À la jonction d'une pratique concrète et d'une réflexivité sociale constamment transformée, de l'intérieur, par les «procès d'apprentissage » à l'œuvre dans les mouvements politiques, se découvre l'autodétermination d'une communauté démocratique.

$* * *$

(II) Non problématisée par Habermas, existe toutefois une tension au sein du pouvoir communicationnel, entre ses structures universelles «profondes » et l'élément d'anarchie qui le caractérise. Au fondement de l'espace public, se tient une certaine forme de rationalité discursive : celle-ci n'est pas fondée à un niveau strictement transcendanta ${ }^{18}$, et elle ne constitue pas, nous prévient Habermas, un paradigme englobant. Il s'agit plutôt d'une structure qui intègre différents types de rationalité. Quoiqu'il en soit, on a affaire à une conception compréhensive de la raison, qui pose celle-ci au fondement du déploiement politique de l'espace public, et qui constitue le critère à partir duquel s'opère la critique des pathologies de la communication. L'idée, c'est qu'en dernière instance la formation rationnelle de l'opinion et de la volonté à l'œuvre dans l'espace public constitue bien la pierre d'angle de la légitimité du pouvoir. Or, qu'une telle perspective soit dépliée à partir de la pensée arendtienne est problématique. Il importe de dégager le cœur de la confrontation manquée entre Habermas et Arendt, afin de dégager le «non-voir» structurel, pour parler en termes althussériens, qui commande la réappropriation d'Arendt par Habermas.

Ce non-voir a une raison : Habermas part du présupposé selon lequel la thématisation par Arendt de l'espace public répond au souci de penser la légitimité du pouvoir, d'en faire le ressort normatif à partir duquel évaluer les formes pathologiques de domination ${ }^{19}$. Ce postulat initial ne lui permet pas de saisir la spécificité de l'approche arendtienne, qui s'inscrit délibérément à contre-courant de la tradition de la philosophie politique

16. J. Habermas, «Trois versions de la démocratie libérale », Le Débat, 2003/3, n 125 , p. 123 .

17. La question de savoir si la rationalité systémique est véritablement susceptible d'être régulée en dernière instance par les institutions démocratiques n'obtient pas une réponse définitive. Dans sa réponse à la critique de W. Streeck (Gekaufte Zeit), J. Habermas reconnaît l'optimisme néo-keynésien qui imprègne sa théorisation de la régulation économique par l'État-social. Voir «Demokratie oder Kapitalismus?», Blätter für deutsche und internationale Politik, 2013/5.

18. J. Habermas refuse de qualifier son entreprise de «fondationnaliste ». Voir K. O. Apel, Penser avec Habermas contre Habermas, Paris, Éclat, 1992.

19. A. Honneth reconduit cette lecture, et voit en Arendt une ressource pour la philosophie sociale. Voir A. Honneth, «Les pathologies du social», La société du mépris. Vers une nouvelle théorie critique, Paris, La Découverte, 2006. 
et pense le pouvoir à un autre niveau. Il faut ainsi remarquer que la notion d'espace public est relativement sous-déterminée dans la théorie arendtienne, qu'elle ne joue absolument pas le même rôle dans la structure d'ensemble de sa pensée. L'idée d'un «public space», ou d'un «public realm» («domaine public»), pose la publicité, au sens de la visibilité publique, comme une condition de l'action politique. Cette sobre définition, qui indexe le domaine public au royaume matériel des apparences, des phénomènes politiques dont la théorie cherche à dégager les articulations propres $^{20}$, ne le reconduit pas à un degré supérieur de profondeur. Ce qui importe, et c'est ici que la lecture intensive d'Habermas échoue à penser le plan effectif où se déploie le concept arendtien, c'est le champ d'extension de la politique, constamment confrontée à ses limites propres. Si la politique s'articule aussi étroitement à l'espace public, c'est parce qu'elle est précisément dépourvue de fondement : elle s'actualise, dynamique, dans un rapport constant à ses frontières, aux éléments qui la nient ou la menacent. C'est en effet au voisinage de la violence ${ }^{21}$ que la politique se constitue comme telle.

Dès lors qu'on change de sol conceptuel, on est à même de reformuler le problème qui occupe une place centrale chez Arendt: la question de la genèse de la politique, c'est-à-dire d'une pratique collective concrète qui vise à la constitution de rapports humains. Cette question est nécessairement historique, car les modalités de cette pratique varient dans le temps: en fonction des valeurs portées par une collectivité, son sens spécifique de la politique ; mais également en fonction des problèmes qui se posent dans un moment et un espace déterminé. La politique ne peut se penser qu'à l'intérieur d'un agencement territorial et historique. Mais par ailleurs, cette pratique ne saurait être historicisée jusqu'à abolir sa différence spécifique, qui la distingue, en tant qu'elle recouvre l'action, des pratiques de l'œuvre et du travail ${ }^{22}$.

En 1972, Arendt entérine un déplacement important : en dénonçant la confusion entre pouvoir et domination ${ }^{23}$, il s'agit de dégager les coordonnées d'un nouveau paradigme pour penser la politique. Non plus, selon le modèle $\mathrm{du}$ «gouvernement», qui trouve son expression historique la plus déterminée dans l'interprétation utilitariste qu'en fait la bourgeoisie ${ }^{24}$, comme l'objet d'une accumulation de pouvoir dans les appareils de l'État ;

20. «La pensée politique ne peut que suivre le mode d'articulation des phénomènes politiques eux-mêmes et reste tributaire des apparences dans le domaine des affaires humaines », H. Arendt, « De la révolution », L’humaine condition, Paris, Gallimard, Quarto, 2012, p. 338.

21. Voir V. Gérard, « Politique et violence selon Hannah Arendt. La violence antipolitique vs la politisation violente des rapports humains », in G. Sibertin-Blanc (dir.), Violences : philosophie, anthropologie, politique, Toulouse, Europhilosophie éditions, 2010.

22. H. Arendt, «Condition de l'homme moderne », L'humaine condition, op. cit., p. 77.

23. Id., « Du mensonge à la violence », L'humaine condition, op. cit., p. 935 et suivantes.

24. H. Arendt, « L’impérialisme», L'humaine condition, op. cit., p. 384 et suivantes. 
mais, en vertu d'une lecture souterraine à la tradition, nécessitant d'être réactualisée, comme un mode de constitution dynamique de rapports, comme une pratique ou un exercice collectif de l'action. Il n'y a donc pas d'ontologie du pouvoir chez Arendt, précisément parce qu'elle récuse l'identification d'une pratique à une essence qui serait susceptible d'en déterminer le sol. En revanche, le pouvoir a bien une assise phénoménologique, car il s'articule à un certain type de phénomènes qui relèvent de la pratique de l'action.

Il n'y aurait donc pas nécessairement un contre-sens à vouloir extraire de la pratique du discours et de l'acte les structures formelles, entre autres langagières, qui en constituent les contours. En revanche, cette pratique ne saurait en aucun cas être identifiée, en dernière instance, à une figure de la rationalité qui constituerait le dernier mot de la politique. Car l'action politique, en tant qu'elle est élucidée par la notion de pouvoir, c'est-à-dire explicitée en son sens proprement constructif - voire constructiviste ${ }^{25}$, ne renvoie pas à un invariant anthropologique. Sa condition de possibilité est minimale, c'est le seul fait de la pluralité humaine. On ne gagne donc rien à indexer le pouvoir arendtien à un fondement ; c'est précisément ce caractère infondé qui explique l'élément d'une indétermination radicale : principe anarchique, au sens où le «commencement» qu'il annonce n'est pas déjà normé, quoique, s'entrelaçant immédiatement à des dispositifs normatifs, institutions ou valeurs éthiques, il motive la construction de nouvelles normes. Toute la question est de déterminer le rapport entre le niveau proprement politique des espaces publics et les agents qui œuvrent à leur création, c'est-à-dire l'articulation entre la lutte pour l'extension d'une expérience collective propre ${ }^{26}$ et l'identité (les intérêts communs) sur laquelle fait fond la bataille politique. Ce rapport ne peut s'élucider que politiquement - non socialement. En terrain arendtien, la société est en effet le nom d'un processus de naturalisation des rapports humains, qui vise à annihiler la dimension politique de l'action. Le membre de la société ne peut que défendre des intérêts déjà constitués, ceux qui sont censés être les siens du fait de sa situation dans un ordre fonctionnel, qui n'est plus même celui d'une société de classes, reposant structurellement sur des principes politiques. C'est l'ordre antipolitique de la reproduction de l'espèce humaine. Derrière la socialisation de masse, se cache la dé-politisation des rapports, l'annihilation des particularités identitaires, la perte des dynamiques propres à la politique que sont l'alliance,

25. Voir É. Balibar, «Impolitique des droits de l'homme. Arendt, le droit aux droits et la désobéissance civique », Erytheis, n $^{\circ}$ 2, Nov. 2007, p. 62 : Arendt «ne se contente pas de rapporter à l'institution la source du droit positif, mais elle y voit une construction de l'humain comme tel [...]. Toute construction politique implique une combinaison avec l'élément contraire (que nous pouvons dire "impolitique", donc - au moins virtuellement, une recréation permanente du politique à partir de sa propre dissolution) ».

26. Ainsi, le rôle politique et révolutionnaire du mouvement ouvrier s'explique par le fait qu'il a cherché à «fonder, au moins en son sein, un nouvel espace public doté de normes politiques nouvelles ». H. Arendt, L'humaine condition, op. cit., p. 256. 
l'accord, l'association - figures diverses de constitution d'une civilité qui émerge au voisinage étroit de la violence qui la menace, et qui en infléchit nécessairement, de fait, le contenu.

On voit par là que la formation rationnelle de la volonté n'est certainement pas le fondement de l'espace public arendtien. Non pas au sens où celui-ci serait à penser selon le modèle de l'agôn, et non de la délibération $^{27}$; mais parce que l'idée selon laquelle l'intersubjectivité livrerait les structures intégratives d'une forme homogène, et non historiquement déterminée, de rationalité, est aux antipodes de la compréhension arendtienne du pouvoir. Parce que celui-ci est aux prises avec la violence, susceptible de le menacer en sa condition de possibilitée $^{28}$, sa genèse est à rapporter à l'agencement institutionnel, idéologique, éthique, auquel il doit sa forme. Résistance et limite à la violence, courant le risque indéfini de succomber à sa tentation, le pouvoir ne peut se revendiquer d'aucune norme qui en garantirait la pureté ${ }^{29}$.

À la toute fin de l'article, comme en un retournement paradoxal, Habermas reconnaît, sans le dire, la limite de son interprétation d'Arendt. Revenant à la figure centrale de «l'accord » (Verständigung), sous laquelle il subsume son concept de pouvoir, il admet qu'on ne saurait le concevoir dans les termes d'une formation rationnelle de la volonté. La faute en est selon lui au fait qu'Arendt n'a pas saisi la spécificité d'une rationalité qui n'est pas synonyme d'évidence discursive, mais bien, selon une compréhension faillibiliste, prétention à la validité susceptible d'être critiquée et justifiée. Admettant donc que le fondement du pouvoir arendtien ne saurait résider dans la rationalité du discours, Habermas le cherche dans l'idée de la promesse. Il voit en celle-ci la réminiscence jusnaturaliste de la figure du contrat ${ }^{30}$. Pourtant, la promesse a ceci de

27. Cette distinction de S. Benhabib («Models of Public Space: Hannah Arendt, the Liberal Tradition and Jürgen Habermas », dans C. Calhoun (ed.), Habermas and the Public Sphere, Cambridge, Mass., MIT Press, 1992) ne nous semble pas aller au fond du problème, car elle réduit la notion d'espace public chez Arendt et Habermas au même statut théorique : celui de révéler un «modèle » de la démocratie. C'est supposer - à tort selon nous - que les théories en présence se construisent selon le même champ de forces, consistant à faire de l'espace public le critère de détermination d'une forme spécifique de gouvernement ou d'autoorganisation.

28. C'est la forme prise par la violence dans les régimes totalitaires, lorsqu'elle atteint le seuil de la terreur.

29. C'est pourquoi la question de la « légitimité » du pouvoir est d'emblée exclue par Arendt : le pouvoir est par essence légitime, car il en est la source ultime et lui correspond. En revanche, la justification du pouvoir implique le recours à des principes extérieurs : ceux-ci peuvent être des principes de justice. C'est l'enjeu de la pensée de chercher à les déterminer (Voir H. Arendt, La vie de l'esprit).

30. J. Habermas, Politisch-philosophische Profile, op. cit., p. 248 : Arendt « sucht nach einer anderen Grundlage für die Macht der Meinung und findet sie in dem Vermögen sprach- und handlungsfähiger Subjekte, Versprechen zu geben und zu halten [...]. Um den normativen 
spécifique selon Arendt qu'elle donne au pouvoir mesure, unité et cohérence, face à la violence que la politique suspend ${ }^{31}$. Là encore, loin de la pensée contractuelle classique, elle demande à être relue dans le rapport entre pouvoir et violence. C'est ce rapport en effet qui permet de réfléchir l'élément de contingence portée par la genèse du politique, élément exclu de la théorisation habermassienne du pouvoir communicationnel.

\section{$* * *$}

En présentant ainsi le problème d'une rationalité propre à l'espace public politique, il s'est agi pour nous, non de repenser en termes contextuels l'universalisme habermassien, mais d'interroger une autre genèse de la politique, qui ne soit pas suspendue à ses conditions de possibilité transcendantales. D'une genèse qui prenne en charge l'extériorité où prend corps la politique, à savoir la contingence, historique, à la fois déterminée et aléatoire, qui explique le caractère fondamentalement imprévisible de l'événement. Cela permettrait peut-être de penser cette rationalité dépourvue de fondement, et pourtant non démunie ${ }^{32}$, que A. Wellmer a appelé de ses vœux.

\footnotetext{
Kern einer ursprünglichen Äquivalenz, zwischen Macht und Freiheit zu sichern, vertraut sie am Ende der ehrwürdigen Figur des Vertrages mehr als ihrem eigenen Begriff einer kommunikativen Praxis. So tritt sie in die Tradition des Naturrechts zurück» (Nous traduisons : Arendt « cherche un autre fondement au pouvoir de l'opinion et le trouve dans la faculté des sujets capables de parler et d'agir, à faire des promesses et à les tenir [...]. Afin d'assurer le contenu normatif d'une équivalence originaire entre le pouvoir et la liberté, elle finit par témoigner davantage de confiance à la figure respectable du contrat qu'à son propre concept de praxis communicationnelle. C'est ainsi qu'elle fait retour à la tradition du droit naturel »).

31. H. Arendt, The Human Condition, Chicago, Univ. of Chicago Press, 1958, p. 200 : «Power is actualized only where word and deed have not parted company, where words are not empty and deeds not brutal ».
}

32. A. Wellmer, «Die Bedeutung der Frankfurter Schule heute», Endspiele: Die unversöhnliche Moderne, Francfort, Suhrkamp, 1993, p. 234. A. Wellmer y revendique « eine bodenlose und doch nicht hilflose Vernunft ». 